\title{
Intracranial Malignant Glioma Presenting as Subarachnoid Hemorrhage
}

\author{
Stephen Hentschel, Brian Toyota
}

\begin{abstract}
Objective: Cerebral aneurysms are the predominant cause of spontaneous subarachnoid hemorrhage (SAH). However, if an aneurysmal cause has been excluded, there remains but a short list of other potential etiologies. Cerebral neoplasms are clearly on this list but are most commonly meningiomas or metastatic lesions. This article details a case of a neoplasm that presented exclusively with SAH. Clinical Presentation: A 40-year-old male presented with a SAH with normal cerebral angiography. The initial magnetic resonance image revealed a lesion in the right insula thought to be resolving hemorrhage. Subsequent images, however, revealed the mass to be enlarging. Intervention: Craniotomy and resection of the lesion established a diagnosis of a malignant oligodendroglioma. Conclusion: An affirmation is made that patients experiencing 'angiographically-negative' SAH should undergo MRI, occasionally on a serial basis, to exclude other etiologies for hemorrhage, including neoplasia.
\end{abstract}

RÉSUMÉ: Hémorragie sous-arachnö̈dienne comme première manifestation d'un gliome malin intracrânien. Objectif: Les anévrismes cérébraux sont la cause première d'hémorragie sous-arachnoïdienne spontanée. Cependant, si une cause anévrismale a été exclue, un petit nombre d'autres étiologies potentielles doivent être considérées, dont les néoplasies cérébrales. Parmi celles-ci les plus fréquentes sont les méningiomes et les lésions métastatiques. Cet article présente un cas de néoplasie dont la présentation initiale était une hémorragie sous-arachnoïdienne sans autre manifestation clinique. Présentation clinique: Un homme âgé de 40 ans a présenté une hémorragie sousarachnoïdienne avec une angiographie normale. L'imagerie par résonance magnétique (IRM) a montré une lésion dans l'insula droite qu'on a pensé être une hémorragie en voie de résolution. Des images subséquentes ont cependant montré une augmentation de volume de la masse. Intervention: La résection de la lésion par crâniotomie a montré qu'il s'agissait d'un oligodendrogliome malin. Conclusion: Les patients qui présentent une hémorragie sousarachnoïdienne et dont l'angiographie est négative devraient subir une IRM sériée au besoin, pour exclure d'autres étiologies de l'hémorragie, dont une néoplasie.

Can. J. Neurol. Sci. 2002; 29: 63-66

The patient who presents with a subarachnoid hemorrhage (SAH) will typically have a cerebral aneurysm found on angiography. However, the cooperative study reported that $43 \%$ of SAHs had an etiology other than cerebral aneurysm. ${ }^{1}$ In these instances, angiography may reveal an arterio-venous malformation or arterio-venous dural fistula. Furthermore, in the cooperative study, an estimated $27 \%$ of angiograms performed to investigate $\mathrm{SAH}$ were normal. ${ }^{1}$ Of the various nonaneurysmal causes of SAH, brain tumors, in the adult population, represent 1 $-3 \%$ of cases. ${ }^{1,2}$

A small volume glioma that presented with a SAH is of interest for its unique presentation, the subtle findings initially seen on magnetic resonance imaging (MRI), and the subsequent evolution of the lesion. The objectives of this case report are to describe the case of an oligodendroglioma as the cause of SAH, to heighten the awareness of neoplasia as a possible cause of angiographically negative $\mathrm{SAH}$, and to outline the importance of further investigation using MRI.

\section{CASE Report}

A 40-year-old male presented acutely with paresthesia involving the left face, arm, and hand. This was followed rapidly by a generalized seizure. He was intubated and ventilated at the scene by ambulance personnel who found him unconscious but not actively seizing.

Over the next several hours his level of consciousness and a mild post-ictal left hemiparesis improved rapidly and he was extubated. He continued to experience a severe headache. A CT scan performed just after arrival in hospital demonstrated SAH involving both Sylvian fissures, with a greater amount of blood in the right fissure and insula and the interhemispheric fissure (Figure 1). The CT scan did not reveal

From the Department of Surgery, Division of Neurosurgery, Vancouver Hospital and Health Sciences Centre, University of British Columbia, Vancouver, BC Canada. ReCEIVED JANUARY 30, 2002. ACCEPTED IN FINAL FORM JUNE 12, 2002.

Reprint requests to: Brian Toyota, Department of Surgery, Division of Neurosurgery, 910 West 10th Ave, 3rd Floor, Vancouver, BC V5Z 4E3 Canada. 
any intra-axial abnormalities. A complete and technically satisfactory four-vessel angiogram was performed but did not reveal a source of bleeding. Furthermore, there was no vasospasm, arterial branch occlusion, or venous obstruction. Examination of the external carotid cranial vessels, in search of a dural fistula, was also noncontributory.

An MRI was performed on the third day of admission that demonstrated a $1 \mathrm{~cm}$ lesion in the right posterior Sylvian fissure that was hyperintense on proton density and $\mathrm{T} 2$, consistent with extracellular methemoglobin. There was minimal surrounding edema. Given the clinical scenario, the appearance on the MRI sequences was felt to be most consistent with a vascular malformation that had hemorrhaged, such as a cavernoma (Figure 2). One week after presentation, three days after his initial angiogram, the patient underwent a second angiographic study. A vascular cause for the SAH was still not found. The working diagnosis at the time was a SAH secondary to a right insular cavernoma. The seizures were controlled with anti-epileptic medication and the patient was otherwise asymptomatic and had no neurologic deficits. He was discharged home with plans for follow-up outpatient MRI and clinical re-assessment.

Two months later he had a repeat MRI scan which displayed hyperintensity in the posterior aspect of the right Sylvian fissure on proton density and $\mathrm{T} 2$ weighted images. Other sequences were not performed at this time. This was interpreted to be consistent with the appearance of a resolving hematoma. The patient remained neurologically intact.

A third MRI scan was performed five months after the initial ictus, and demonstrated similar findings to the previous. At eight months after initial presentation the patient again experienced a partial seizure localized to the right hemisphere as well as increasing headache. An MRI scan on this occasion showed the lesion to have increased in size by $1 \mathrm{~cm}$ and was associated with patchy and inhomogeneous contrast enhancement with little surrounding edema (Figure 3). Despite the initial presentation of SAH, the serial magnetic resonance images made glioma the most likely diagnosis.

Using neuro-navigation and intra-operative motor mapping, a craniotomy and partial resection of the tumor was performed. Outside of remote bloodstaining of the overlying meninges, the brain surface itself appeared normal. There was no abnormal vasculature. The tumour itself was only moderately, and not unusually, vascular. Pathology revealed a malignant glioma remarkable for pleomorphism, mitoses, necrosis, vascular neogenesis, and neuronal satellitosis. The glial component was largely oligodendroglial but also contained some astrocytic elements. Final pathology was that of an anaplastic oligodendroglioma with a lesser component of astrocytoma. The patient went on to receive 60Gy in 30 fractions of external beam radiotherapy followed by adjuvant chemotherapy consisting of four cycles of Procarbazine, CCNU, and Vincristine.

At 17 months since original presentation and nine months after craniotomy the patient remains neurologically intact with complete seizure control on medication. The tumor itself appears stable on MRI at nine months after surgical resection.

\section{Discussion}

Nonaneurysmal cases of SAH represent a substantial portion of a neurosurgeon's patient population. The concept of the perimesencephalic SAH has become well-entrenched as a verifiable phenomenon. ${ }^{3}$ Although its exact anatomic substrate remains elusive, the clinical course and prognosis are reasonably wellestablished. ${ }^{4}$ The other nonaneurysmal causes for SAH include coagulopathy, hypertension, infection, venous occlusive disease, occult trauma, and cerebral neoplasia. ${ }^{5}$

A brain tumor as the cause of SAH is not typically a source of uncertainty as the lesion is usually strongly suggested, if not well
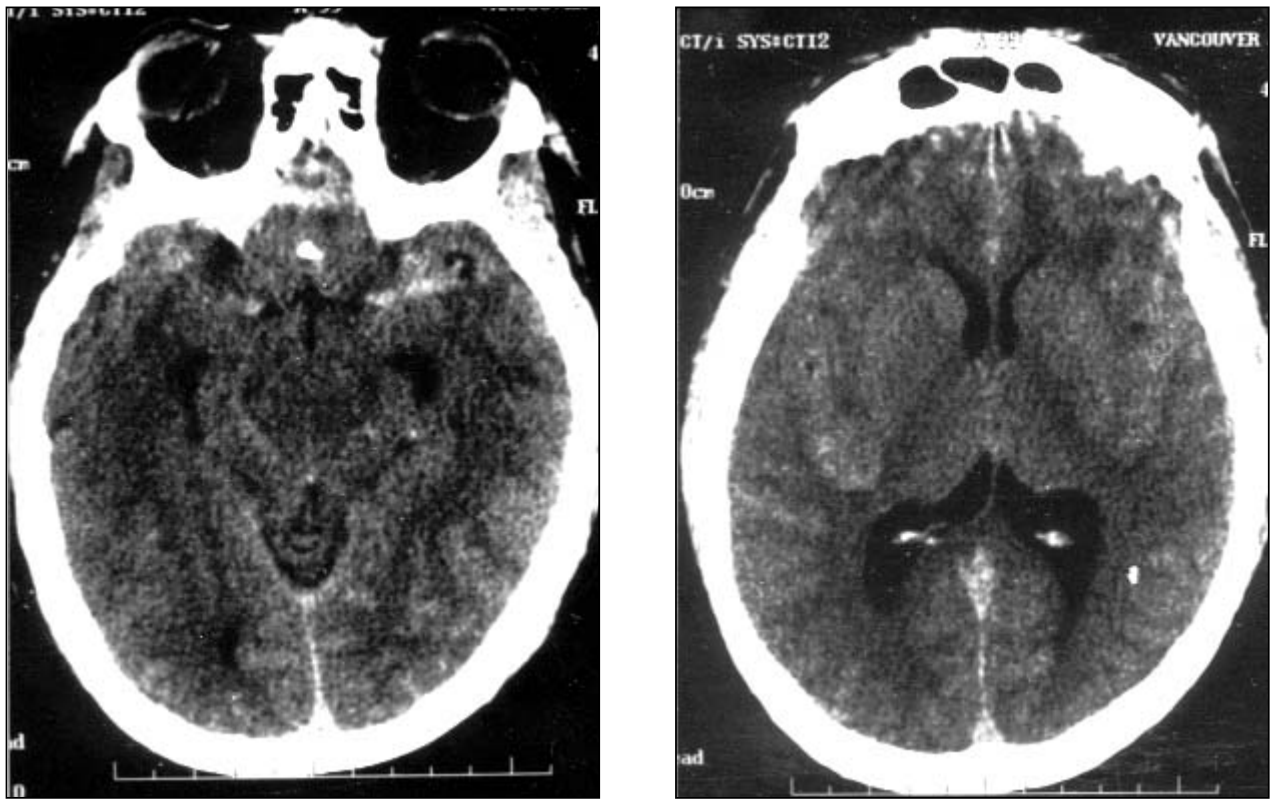

Figure 1: Nonenhanced CT scans demonstrating acute subarachnoid hemorrhage involving a) the Sylvian fissures bilaterally as well as the basal cisterns and b) the right insular region. Unfortunately, the images are degraded by patient motion. 

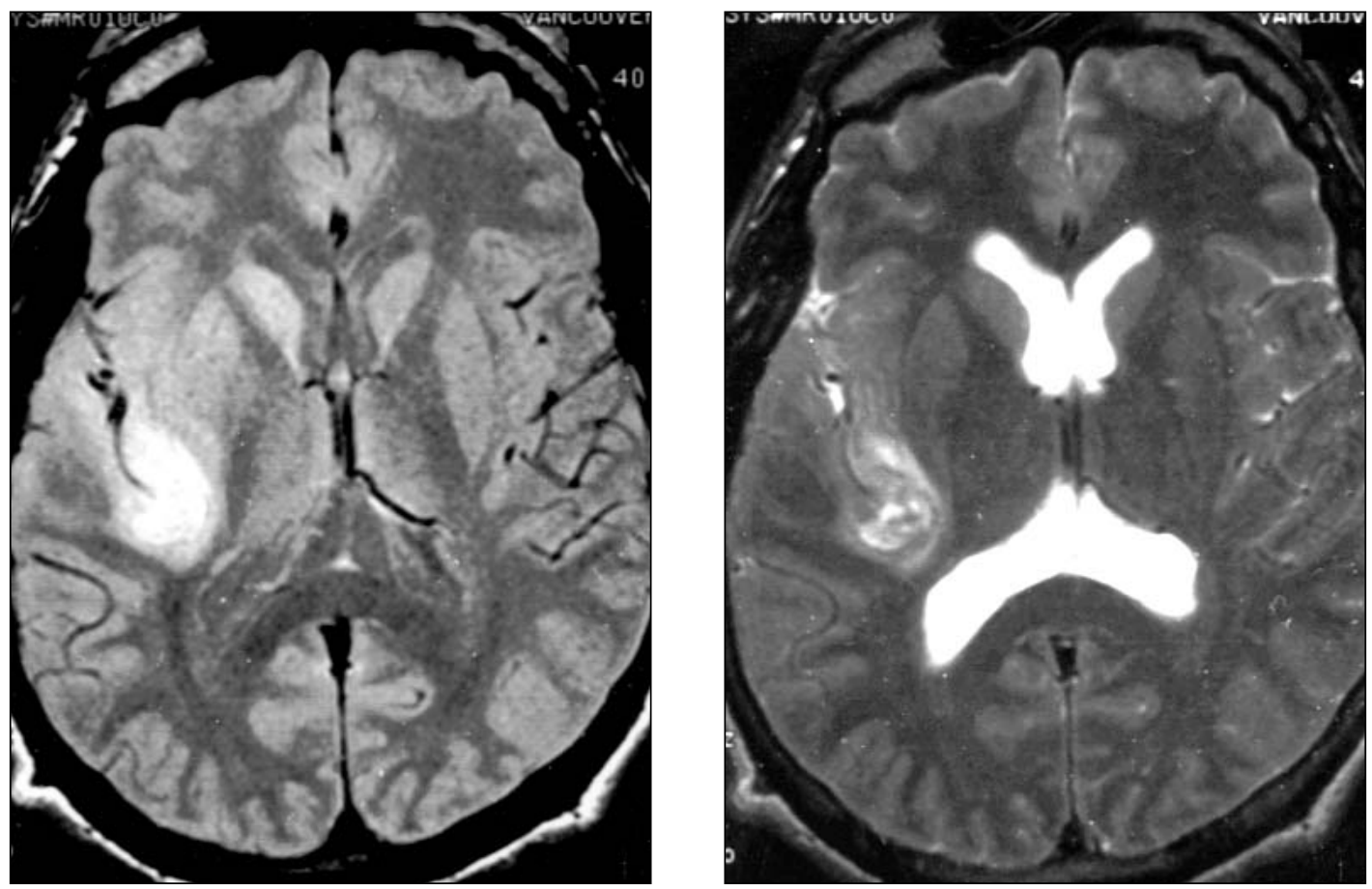

Figure 2: MRI at presentation: a) axial proton density (TE 32, TR 2000) and b) axial T2 (TE 85, TR 4000) sequences demonstrating high signal.
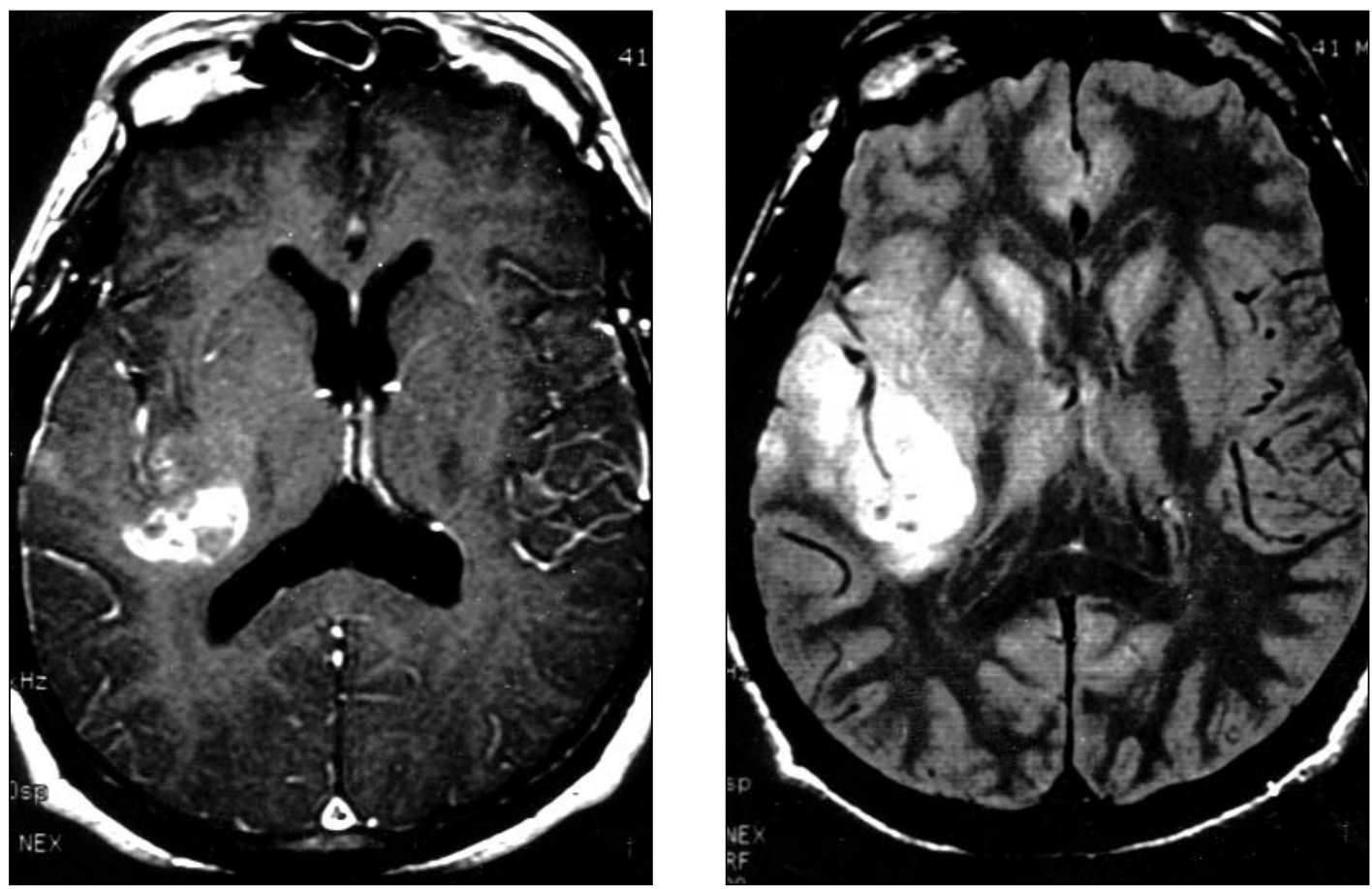

Figure 3: MRI at five months after presentation; a) Enhanced axial T1 (TE 24, TR 600) and b) axial proton density (TE 32, TR 2000) sequences demonstrating an inhomogeneously enhancing lesion in the right insula. 
depicted, on initial CT imaging. In these cases, the literature consistently reports meningiomas ${ }^{6,7}$ and cerebral metastatic lesions ${ }^{8,9}$ (particularly melanoma, choriocarcinoma, renal cell carcinoma, and thyroid carcinoma) as the underlying neoplasm. Although gliomas have a significant hemorrhage rate, they are almost exclusively intra-parenchymal bleeds, with only rare extension to the subarachnoid space. ${ }^{10,11}$ Due to the high frequency of glioblastoma, it is more common to see hemorrhage with glioblastomas than with oligodendrogliomas, however, the relative frequency of hemorrhage is higher in oligodendroglioma than with glioblastoma. ${ }^{12}$ Up to $30 \%$ of cases of oligoastrocytomas may present with evidence of macroscopic hemorrhage. ${ }^{13}$ Particularly in the scenario of an underlying meningioma or metastases with a known systemic cancer, the original investigative CT scan may often preclude angiography. However, a glioma as the source of SAH is not a common consideration. The underlying pathophysiology has been understood to involve a tenuous and necrotic neovasculature, neoplastic invasion of nascent cerebral vessels, and the propagation of a systemic coagulopathy. ${ }^{14,15}$ What makes this case unique is the seemingly occult nature of the lesion and the isolated hemorrhage into the subarachnoid space.

Several considerations are required for the patient with a SAH who has an initial angiogram that does not reveal a hemorrhagic source. Assurances must be made that a complete four vessel study has been made, with particular attention paid to the visualization of both posterior inferior cerebellar arteries. Arterial bifurcations or infundibuli must be imaged from various angles to ensure that small aneurysms are not overlooked. Areas of arterial stenosis and spasm should be looked for as hints towards 'hidden' aneurysms. Once an aneurysm has been definitively excluded, other vascular causes of SAH must be considered. Analysis of the angiogram must include a search for early draining veins suggestive of small arterio-venous malformations, dissecting arterial branches and external carotid angiography to evaluate arterial-venous dural fistula. There remains debate regarding the value of repeat angiography in this setting. Reports of false-negative initial angiograms range from $2-22 \% .{ }^{16-18}$ Current practice often leads to repeat angiography for that patient with clear radiologic evidence of SAH and any equivocation regarding the initial angiogram. Finally, one must be wary of spinal vascular lesions that cause intracranial $\mathrm{SAH}$, and are often missed without astute clinical acumen. ${ }^{19}$

If the $\mathrm{SAH}$ has been definitively diagnosed as nonaneurysmal, then there remains a short list of differential diagnoses that must be investigated. The majority can be diagnosed by concise history-taking and neurologic exam, standard and specific blood work (such as coagulation profiles and complete blood count in particular), and CT imaging. The role of MRI is less clear as few studies have examined the utility of MRI in investigating this group of patients. One study of 30 patients with 'angiogram-negative' SAH who were subsequently investigated with MRI, found two positive studies. ${ }^{20}$ One patient was identified as having a previously unrecognized aneurysm (also seen on follow-up angiography) and another was found to have a brainstem lesion of uncertain significance. Magnetic resonance imaging has three definitive differentials to evaluate: a thrombosed aneurysm, a cavernoma and, as this case documents, a small glial neoplasm. Given these possibilities, we suggest that MRI become a standard investigation for the patient with an 'angiogram-negative' SAH.

\section{Conclusions}

In presenting this case we have added malignant oligodendroglioma to the differential in the case of nonaneurysmal SAH and made the argument towards MRI as routine investigation in the scenario of 'angiogram-negative' SAH.

\section{REFERENCES}

1. Locksley H, Sahs A, Sandler R. Report on the cooperative study of intracranial aneurysms and subarachnoid hemorrhage III. Subarachnoid hemorrhage unrelated to intracranial aneurysm and AV malformation. A study of associated diseases and prognosis. J Neurosurg 1966;24:1034-1056.

2. Courville C, (ed). Pathology of the central nervous system. Mountain View, CA: Pacific Press Publishing Association; 1937.

3. Van Gijn J, Van Dongen $K$, Vermeulen $M$, Hijdra A. Perimesencephalic hemorrhage: a nonaneurysmal and benign form of subarachnoid hemorrhage. Neurology 1985;35:493-497.

4. Rinkel G, Wijdicks E, Hasan D, et al. Outcome in patients with subarachnoid haemorrhage and negative angiography according to pattern of haemorrhage on computed tomography. Lancet 1991;338:964-968.

5. West H, Mani R, Eisenberg R. Normal cerebral arteriography in patients with spontaneous subarachnoid hemorrhage. Neurology 1977;27:592-594.

6. Askenasy H, Behmoaram A. Subarachnoid hemorrhage in meningiomas of the lateral ventricle. Neurology 1960;10:484-489.

7. Helle T, Conley F. Hemorrhage associated with meningioma: a case report and review of the literature. J Neurol Neurosurg Psych 1980;43:725-729.

8. Dagi TF, Maccabe JJ. Metastatic trophoblastic disease presenting as a subarachnoid hemorrhage: report of two cases and review of the literature. Surg Neurol 1980;14:175-184.

9. Spetzger U, Mull M, Sure U, Gilsbach J. Subarachnoid and intraventricular hemorrhage caused by hypernephroma metastasis, accompanied by innocent bilateral posterior communicating artery aneurysms. Surg Neurol 1995;44:275-278.

10. Glass B, Abott K. Subarachnoid hemorrhage consequent to intracranial tumors. Arch Neurol Psych 1955;73:369-379.

11. Oldberg E. Hemorrhage into gliomas: a review of eight hundred and thirty-two consecutive verified cases of glioma. Arch Neurol 1933;30:1061-1073.

12. Batjer H, Kopitnik TA, Friberg L. Spontaneous Intracerebral and Intracerebellar Hemorrhage. In: Youmans J, editor. Youmans' Neurological Surgery. W.B. Saunders Company; 1997.

13. Kondziolka D, Bernstein M, Resch L, et al. Significance of hemorrhage into brain tumors: clinicopathological study. J Neurosurg 1987;67:852-857.

14. Liwnicz BH, Wu SZ, Tew JM Jr. The relationship between the capillary structure and hemorrhage in gliomas. J Neurosurg 1987; 66(4):536-541

15. Nutt $S$, Patchell R. Intracranial hemorrhage associated with primary and secondary tumors. Neurosurg Clin N Am 1992;3:591-599.

16. Susuki S, Kayama T, Sakurai Y. Subarachnoid hemorrhage of unknown cause. Neurosurgery 1987;21:310-313.

17. Perret L, Bull J. Some aspects of subarachnoid hemorrhage: a symposium III. The accuracy of radiology in demonstrating ruptured intracranial aneurysms. Br J Radiol 1959;32:85-92.

18. Foster D, Steiner L, Hakanson S, Bergvall U. The value of repeat panangiography in cases of unexplained subarachnoid hemorrhage. J Neurosurg 1978;48:712-716.

19. Chalif D, Black K, Rosenstein D. Intradural spinal cord tumour presenting as subarachnoid hemorrhage: magnetic resonance imaging diagnosis. Neurosurgery 1990;27:631-634.

20. Renowden S, Molyneux A, Anslow P, Byrne J. The value of MRI in angiogram-negative intracranial hemorrhage. Neuroradiology $1994 ; 36: 422-425$. 